\title{
Application of Machine Vision on the Nut Collars Sort System
}

\author{
LIU Ling-yun, LUO Min, WU Yue-min
}

School of Electric and Information Engineering, Hubei University of Automotive technology,

Shiyan city, hubei province, China

Keywords: dimension detection, machine vision, sort out

\begin{abstract}
Some problems, such as slow speed, inefficient and excessive labour, etc, may exist if the quality detection is carried out artificially in the volume-production of nut collars. Therefore, the machine vision is introduced into the dynamic inspection system, which can automatically measure the inside and outside diameter and proper alignment of the nut collars in the product line. At the same time, this will trigger the sort subsystem to separate the waste products from others. As a result, it achieves the rapid measure of crucial dimension and the arrangement of grades.
\end{abstract}

\section{Foreword}

With the degree of automation requirements improve constantly in recent years, the machine vision as a new technology to be used in industrial, medical, remote sensing detection and intelligent traffic etc[1].One of these,on-line inspection of the products of machine vision is a kind of application most widely.

Elastic nut washer is one of the important parts in the motor vehicle assembly,which should directly affect the pre-tightening force distribution of the automobile parts and components, so it have the strict technical requirements for the gaskets inside and outside round error 、 concentricity error etc during the production. The most enterprises domestic current take quality detection for nut washer with adopting the form of artificial sampling, there being slow speed, large amount of labor and easily omission factor etc.

This paper puts forward a nut washer online detection and automated sorting system based on machine vision.The system has a high speed data acquisition, on-line real-time processing ( dimension Inspection, defective items elimination etc) and display( alarm),it vastly overcomes the defect of manual sampling,and verify the feasibility of the system by the experiment.

\section{The hardware composition of visual sorting system}

Q / 2E185 wave spring washers(Here in after referred to as the washer), which normally adopt the thickness 0.5 millimeter beryllium bronze(QBe2-cy)as the sheet metal, go by baiting $\rightarrow$ stamping $\rightarrow$ deburring $\rightarrow$ crooked $\rightarrow$ heat treatment aging $\rightarrow$ inspection process etc. During the inspection process, in addition to take sampling inspection for the elastic of gasket, also must test gasket dimension, to ensure both inside and outside round error is $\pm 0.1 \mathrm{~mm}$, the concentricity error is $\Phi 0.1$ millimeter.

In order to meet the requirements of customer for high speed, comprehensive detection in the production of the large quantities of gasket, this paper proposes a kind of sorting system based on machine vision, it can detect the gaskets of the automatic line on-line and weed out unqualified products, the system consists of the sorting subsystem、 the transmission subsystem 、 the image acquisition subsystem、 the light source subsystem、 the image processing and analysis subsystem、 the signal control subsystem and eliminate subsystem etc, the whole system frame structure as shown in graphic 1. 


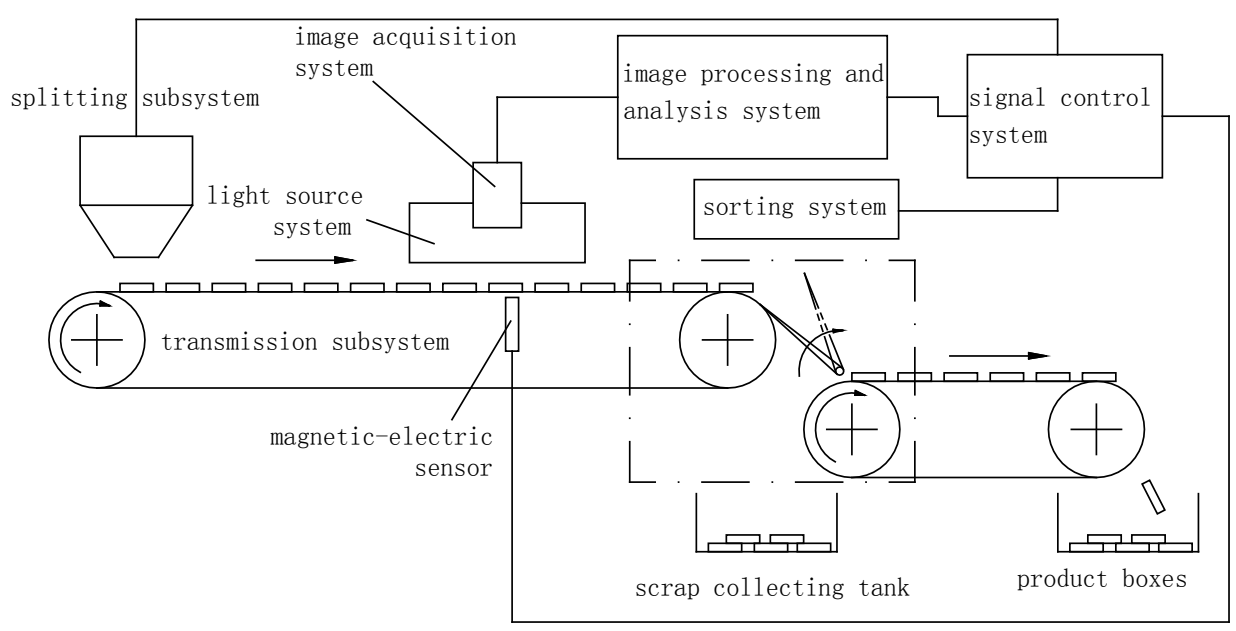

Graphic 1 visual sorting system hardware structure diagram

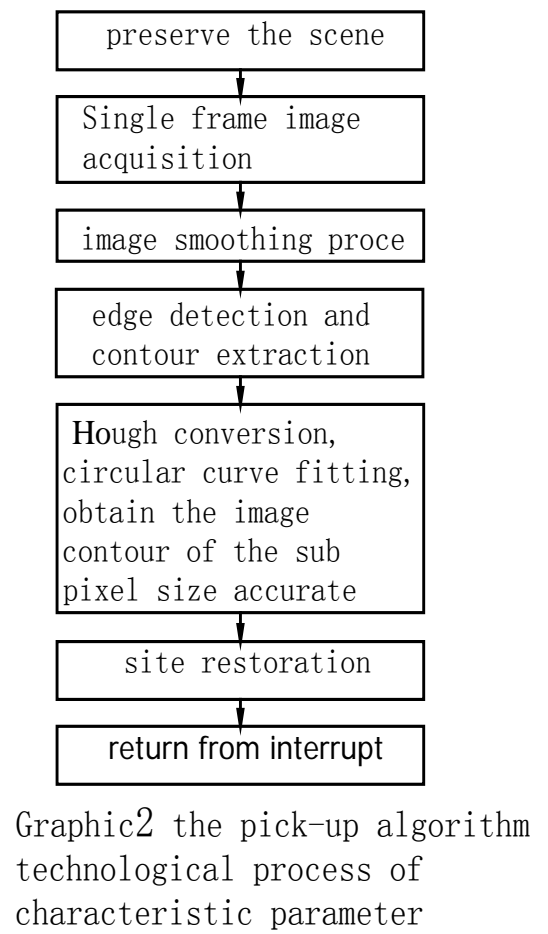

The coordinated action ,between the splitting subsystem and the transmission subsystem, ensure that the gaskets are arranged to the conveyor belt according to certain interval,when the magnetic-electric sensor at the bottom of the camera inspected the trigger signal with the gasket go through nearby place,to trigger the image acquisition subsystem to take photo the gasket at the bottom of the camera,and the collected images were sent to the image processing and analysis subsystem for processing and judging, while to found testing index which be over proof to take the output signal to the eliminate subsystem.The eliminating subsystem will control the movement of the actuator after a period of time delay,the unqualified washers fell into the waste collection containers.

In this system low angle LED lighting light source ,provided LDR2-LA70SW by CCS company was use,which can better display the edge of the nut washer image, improve the contrast of goals and the surrounding environment,reduce the difficulty and stress of image processing late.

The high sensitivity and high resolution CCD camera DH-SV1411FM, provided by DAHENG company,have several working modes as follow: continuous collection 、 external trigger (programmable exposure 、 electrical level exposure)、 soft trigger etc.In order to meet the needs of real-time detection,the camera is set to programmable exposure method in this system,after the camera detected rising edge of the trigger signal external,start to exposure (exposure time be intended is 50 us), to collect a frame images,to transmit to the host controller. In order to prevent the 
interference signal and the damage of the camera,the connection adopt by optical coupling isolation between the sensor and the camera.

\section{The software system structure and main function module}

The function modules of the software system include system initialization 、 image collection and processing, data statistics management etc. In which the initialization subprogram complete this setting of the camera works , the system operation parameters and the initialization of variables;data statistics management subroutine is used for the data testing of the washer and to archive these dada、 finished goods/scrap count management etc; the image collection and processing is the core module of this system, it has a strong real-time performance,to complete the corresponding functions in the interrupt service subroutine external sensor trigger,the interrupt subroutine block diagram is shown in graphic 2 .

\section{Image smoothing processing.}

The images gathered by this collection system suffer easily interference with the blot on parts 、 the stain on lens, impurities in the air , the electromagnetic radiation by irradiation light and electronic device etc,these interference data will be included in the transmitting procedure of the data acquisition front-end or after finishing the collection,bring with the adverse effects of image clear view value[2]. Therefore, we must be worked to the image noise filtering processing before the image processing. This system adopts the $7 \times 7$ median filtering method,this method can reduce nonlinear smoothing of the edge fuzzy, it well can eliminate the impulse noise[3], it can to guarantee the accuracy of image feature extraction later.Collection the original image and the image filtering as shown in graphic $3(\mathrm{a})$, (b).

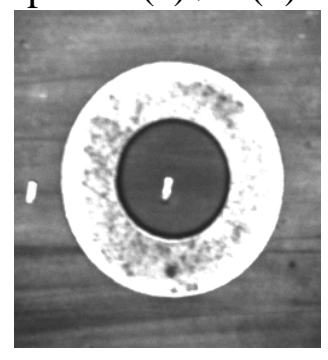

(a) Original image

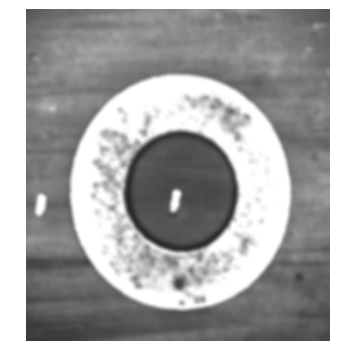

(b) the image after a median filter

Graphic 3 Image smoothing processing

\section{Edge detection and contour extraction.}

In the system software design, to begin with setting gray-level threshold of image binarization processing (Take 150 gray level threshold), and according to the binary morphological operation

(Connected domain area、Edge Detection、Corrosion、Swelling), to extract Contain wait detect edge information area from the image, in order to reduce the computation late image, to improve the response speed of system. The extraction process of ROI as graphic 4（a)、(b)、 (c) shows.

Image edge detection is the premise of nut washer size detection, as much as possible to improve the detection accuracy, this system use Canny operator to extract the edge pixels of nut washer image, smooth filtering, then Adopt "non-maxima suppression" the double threshold operation method, to inhibition of pseudo edge points and to Connect the real point, as show in the graphic 4(d), to utilize finally the edge character for extracting, so getting the edge image required.

After the pretreatment above-mentioned, the images have well inside/outside diameter, for preparing for the next step size measurement. The result of image preprocessing as show in graphic 4(e). 


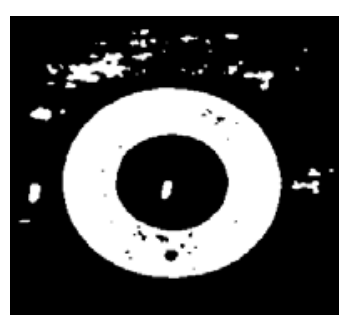

(a) Binarization processing

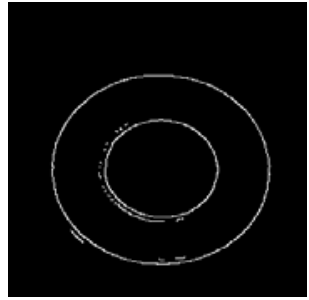

(d) Canny operator process image

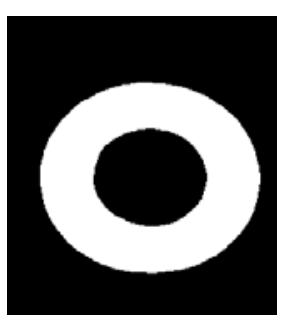

(b) morphological algorithm

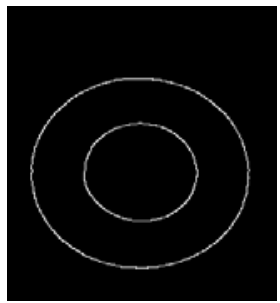

(e) edge extraction

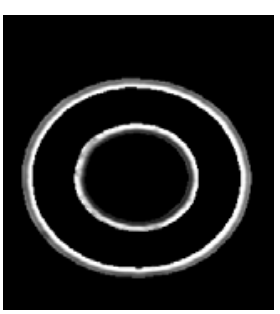

(c) ROI

Graphic 4 the process of edge detection and contour extraction

\section{The measurement of gasket image geometry size( sub-pixel accurate)}

With its high reliability and good characteristics fault tolerance and robusmess in the regional boundary noise interference or covered with other goals thus cause the some disconnected cases of the boundary , Hough transform have been widely used in the circle detection. The basic thought is to be using two arbitrary non- parallel strings arbitrary two non- parallel strings the circumference of a circle of arbitrary two non- parallel strings on midperpendicular meet in the nature of the center of the circle, to choose three point on the circumference of a circle at the same time, so the parameters of the circle to be determined.

In allusion to the preprocessed edge image ,firstly, the probability statistics was done from the counted radius $\mathrm{Ri}$ of the circle with the analytic geometry, to found $\mathrm{Ri}$ value used most as the radius value of the circle, and record the pixel coordinate value $\mathrm{P}(\mathrm{xi}$, yi)with corresponding edge points on the circumference of a circle. in accordance with the principle of point Hough transform.At this point the value of the round radius is the pixel level, in order to achieve higher precision sub-pixel detection, to use the least squares principle, for fitting the edge point $\mathrm{P}$ (xi, yi) recorded, to figure out the parameter of circle (radius, center position).

According to the principle of least squares (the sum squared of residuals is minimal), circle was approximated with the circular contour. The equation of circle is as follow.

Hereby, residuals is adopt as:

$$
(x-a)^{2}+(y-b)^{2}=r^{2}
$$

$$
\varepsilon i=(x i-a)^{2}+(y i-b)^{2}-r^{2}
$$

In the equation: $i \in E, E$ is the set of edge point.

The function of sum squared of residuals is :

$$
Q=\sum_{i \in E} \varepsilon_{i}^{2}=\sum_{i \in E}\left[\left(x_{i}-a\right)^{2}+\left(y_{i}-b\right)^{2}-r^{2}\right]^{2}
$$

According to the principle of least squares, should have:

$$
\frac{\partial Q}{\partial a}=\frac{\partial Q}{\partial b}=\frac{\partial Q}{\partial r}=0
$$

By the above equation, the geometric parameters of the circle can be elicited.

Which we should note here is, to solve the radius of round $\mathrm{Ri}$,it should as far as possible make every three edge points are equally distributed throughout the entire circumference, so as to ensure the reliability and accuracy of calculation.

\section{Test Result}

This system adopted the DH - SV1411FM high sensitivity high resolution CCD camera, the imaging element is $2 / 3$ SONY EX-VIEW CCD, imaging element size is 6.45um×6.45um.Using the 
least squares fitting circle and to subdivide the pixels, the measurement precision of radius can reach 0.3 times pixel, the measurement precision of the diameter can reach $3.8 \mathrm{um}$.

In order to improve the calibration accuracy, reduce the system error, to choose less distortion lens M1214 - MP, at the same time using "the colorimetric method" for calibration system,the qualified washer known outside diameter size D as the standard washer,to calculate the surface resolution $d x$ of the cameras $(d x=D / N, N$ is the average value of the diameter pixel values by measurement many times), it be as the calibration value.This method does not need to calibrate the camera's internal parameters, it's simple process, low cost, easy to achieve.The results of diameter calibration are shown in table 1.

Table1 The results of diameter calibration $(\mathrm{N}=20)$

\begin{tabular}{|c|c|c|c|c|c|c|}
\hline $\begin{array}{c}\text { maximum } \\
\text { (pixels) }\end{array}$ & $\begin{array}{c}\text { minimum } \\
\text { value } \\
\text { (pixels) }\end{array}$ & $\begin{array}{c}\text { average } \\
\text { value }\end{array}$ & variance & $\begin{array}{c}\text { standard d } \\
\text { eviation }\end{array}$ & $\begin{array}{c}\text { standard gasket } \\
\text { external diameter } \\
\text { ( mm })\end{array}$ & $\begin{array}{c}\text { image surface } \\
\text { resolution } \\
\text { (mm/ pixels) }\end{array}$ \\
\hline 105.5706 & 105.5276 & 105.551 & 0.000517 & 0.022756 & 38.00 & 0.3600126 \\
\hline
\end{tabular}

Using the calibration value above mentioned test repeatedly for a standard nut washer(Inside and outside diameter:20/38,coaxiality: $\phi 0.08 \mathrm{~mm}$ ) ,the inside and outside diameter measured are: 20+0.045/-0.033mm、38+0.041/-0.039mm;coaxiality: $\phi 0.02 \sim 0.12 \mathrm{~mm}$. So the measuring accuracy of the washer machine vision detection system online make a breakthrough for the limit of pixel level, to realize the non-contact of washer , the needs of online real-time measurement and automatic sorting. The software system slightly modify can be applied in the products testing of the other industries.

\section{References}

[1] ZANG Tie-fei, SHEN Ting-zhi, CHEN Jian-jun, GU Jian-jun. The Application of Impmved Hansdorff Distance and Genetic Algorithm in Image Matehing[J].Journal of Beijing Institute of Technology,2000,20(6):733-737.(in Chinese)

[2] ARTURO P G,VICTOR A R,RAUL ENRIQUE S Y,et a1. Monte Carlo Evaluation of the Hansdorff Distance for Shape Matching[M]. LNCS, 2006, 4225:686-695.

[3] ARTURO P G,VICTOR A R,RAUL ENRIQUE S Y,et a1 : Monte Carlo Evaluation of the Hansdorff Distance for Shape Matching[M]. LNCS, 2006, 4225:686-695.

[4] Zhu Yanjuan, Zhou Laishui, Zhang Liyan,Liu shuang: A Multiscale Method of Contour Matching Based on Hausdorff Distance[J] . China Mechanical Engineering. 2004, 15(17): 1553-1556.

[5] Liu Lingyun, Qian Xin'en: Reformative Approach to Camera Calibration Based on 3D Pattern[J]. Journal of Hubei Automotive Industries Institute, 2008,1:33-36. 